\title{
Did you know that your body has diagnostic capabilities in itself?
}

Keywords: body, diagnostic, capabilities, life, stress, aggravates, hypothetical, patient, drugs

\section{Opinion}

Did you know that your body has diagnostic capabilities in itself? This system works very well to maintain your entire life. You might think of this system as "Your Doctor Within".

Did you also know that "Your Doctor Within" sends out diagnostic signals, ready for reading? Some of these signal can be received by the patient themselves, e.g. pain. Healthcare professionals can read other signals, but alas, few do. This is probably because these signals are emotional and currently not considered by most physicians.

Some advanced doctors are considering "Emotional Stress" and/ or "Pain" as additional Vital Signs. Typically, the only Vital Signs considered are Body Temperature, Pulse Rate (or Heart Rate), Blood Pressure, and Respiratory Rate (and/or Oxygen Saturation).

\section{Currently medical monitors do not measure emotional stress and/or pain}

Please look for this in the future. With a device of this type, the doctor could "see" what is going on inside the patient, whether they can communicate or not. Everyone knows that Emotional Stress aggravates any medical condition. Some say that Negative Stress causes health maladies. With a little extra work by the doctor, the emotion of the patient could also be determined.

Yet, many patients will not talk about their emotions. Both doctors and patients typically do not like to talk about "feelings". Emotions are important, however, since they do affect a person's health, either negatively or positively. This patient-doctor miscommunication results in misdiagnosis. Some hypothetical examples of a misdiagnosis are:

a) Patient comes in with symptoms of fatigue and lack of interest in life. Typically, doctors will diagnose this as depression and treat it with drugs. Yet, what the doctor did not know was that a close family member had just died. The patient was going through normal grieving. Yes, this is a situation to watch by the patient and doctor, but drugs might not be necessary at this time.

b) Patient has high blood pressure and is overweight. Doctors will typically prescribe medication, and this is good, since hypertension is potentially lethal. In some cases, the doctor might also recommend weight loss to improve blood pressure. The patient agrees with the doctor to lose weight. What the doctor does not know is that the patient is angry inside, does not intend to lose weight, and just wants to eat for comfort. The patient just wants to be given drugs to treat the symptoms of being overweight.
Volume 7 Issue 5 - 2018

\section{Tom Garz}

Writer and Inventor, Michigan Technological University, TG Ideas LLC, USA

Correspondence: Tom Garz, Writer and Inventor, Michigan Technological University, TG Ideas LLC, USA, Email tgedeas@gmail.com

Received: May 29, 2018 | Published: November 20, 2018

The examples above show that doctors only see and hear, but not know the emotional state of the patient. Knowing the emotional situation of the patient can help the doctor diagnose and treat the patient. With the right kind of Medical Monitor, misdiagnosis could be prevented.

Doctors and researchers have a wealth of information on what vital signs change with each emotion. These observations could be compiled into an algorithm within the Medical Monitor to differentiate between emotions. For example, if the patient was angry the heart rate, skin conductance, and temperature would rise. Fear shows up as increased heart rate, skin conductance, but a decrease in temperature. Each emotion has its own physiological diagnostic signal or signature. Further research could refine the algorithm to pinpoint the exact emotion better.

With this additional information, the doctor could better determine what is causing your physical symptoms and treat you accordingly.

\section{Takeaways}

a) Each person has a built-in diagnostic system.

b) Healthcare professionals will soon be able to access a patient's internal diagnostic system. This will result in a more accurate medical diagnosis and treatment plan.

c) Technology is catching up to receive the patient's own diagnostic signals. Look in the future for a Medical Monitor that indicates the patient's Emotional State and/or Pain Level.

\section{Acknowledgements}

None.

\section{Conflicts of interest}

Authors declare that there is no conflict of interest. 